\title{
Phytosomal Nanoparticles Preparation of Curcuminoids to Enhance Cellular Uptake of Curcuminoids on Breast Cancer Cell Line MCF-7
}

\author{
Nguyen Van Long ${ }^{1, \#}$, Bui Thi Thu Ha ${ }^{1, \#}$, Anh Vu Tuan ${ }^{1}$, Hoang Van Luong ${ }^{1}$, Nguyen Tung Linh ${ }^{1}$, Thanh Chu Duc', \\ Phung Cao Dai ${ }^{2}$, Chul Soon Yong ${ }^{2, *}$, Chu Van Men ${ }^{1, *, *}$
}

Nguyen Van Long ${ }^{1, \#, ~ B u i ~}$

Thi Thu Ha, ", Anh Vu Tuan',

Hoang Van Luong ${ }^{1}$, Nguyen

Tung Linh', Thanh Chu Duc ${ }^{1}$,

Phung Cao Dai ${ }^{2}$, Chul Soon

Yong $^{2}$, Chu Van Men ${ }^{1, \#, *}$

'Institute of Biomedicine and Pharmacy, Vietnam Military Medical University,

222-Phung Hung Street, Ha Dong District, Hanoi, VIETNAM.

2Laboratory of Pharmaceutics, College of Pharmacy, Yeungnam University, 214-1

Dae-Dong, Gyeongsan 712-749, REPUBLIC OF KOREA.

${ }^{\#}$ These authors contributed equally to this work.

\section{Correspondence}

Chu Van Men

Ph.D, Director, Clinical Trial and

Bioequivalent Testing Centre, Institute

of Biomedicine and Pharmacy, Vietnam

Military Medical University, 222-Phung

Hung Street, Ha Dong District, Hanoi,

VIETNAM

E-mail: chuvanmen@vmmu.edu.vn

\section{Chul Soon Yong}

Laboratory of Pharmaceutics, College

of Pharmacy, Yeungnam University,

214-1 Dae-Dong, Gyeongsan 712-749,

REPUBLIC OF KOREA.

E-mail: csyong@ynu.ac.kr

History

- Submission Date: 30-06-2019;

- Review completed: 22-07-2019;

- Accepted Date: 24-07-2019

DOI : 10.5530/pj.2019.11.163

Article Available online

http://www.phcogj.com/v11/i5

\section{Copyright}

(C) 2019 Phcogj.Com. This is an openaccess article distributed under the terms of the Creative Commons Attribution 4.0 International license.

\begin{abstract}
Objective: Curcuminoids, the bioactive compounds extracted from Curcuma longa consisting of Curcumin (CUR), demethoxycurcumin (DMC) and bisdemethoxycurcumin (BDMC), have shown promising biological effects, including anticancer activity. This study sought to prepare a physically stable phytosomal nanoparticles of curcuminoids (Curs-Phyto) to facilitate uptake of curcuminoids on breast cancer cells line, and further increase the cytotoxicity against cancer cells. Methods: The evaporation combined extrusion technique was employed to prepare phytosomal curcuminoids nanoparticles. The interaction between curcuminoids and phospholipid by a hydrogen bond was confirmed by differential scanning calorimetry (DSC), powder X-ray diffraction (XRD), fourier transform infrared (FT-IR), and ${ }^{1} \mathrm{H}$ nuclear magnetic resonance ( $\left.{ }^{1} \mathrm{H}-\mathrm{NMR}\right)$. Their physicochemical characterizations and stability in simulated gastric and intestinal media were investigated. The effects of Curs-Phyto on MCF-7 cells were evaluated by flow cytometry, MTS assay and cell cycle analysis. Results: We found that the Curs-Phyto were formed at a spherical shape with good size ( $180 \mathrm{~nm})$, a narrow size distribution (PDI < d0.2), high complexation rate $(\sim 87 \%, 95 \%$, and $90 \%$ for BDMC, DMC, and CUR respectively) and high loading capacity of curcuminoids. More importantly, the Curs-Phyto showed the increased cellular uptake and enhanced cytotoxicity against MCF7 cancer cells, compared to free curcuminoids. Conclusion: These results indicated that the phytosome could be a promising oral delivery system for curcuminoids for cancer treatment. Key words: Curcuminoids, Phytosome, Nanoparticle, Cellular uptake, FACS analysis.
\end{abstract}

\section{HIGHLIGHTS}

- A simple and effective evaporation combined extrusion method to prepare curcuminoidsloaded nano phytosome is successfully applied.

- The physically stable phytosomal nanoparticles of curcuminoids were formed at a spherical shape with good size $(\sim 180 \mathrm{~nm})$, a narrow size distribution (PDI $<0.2$ ), high complexation rate $(\sim 87 \%, 95 \%$, and $90 \%$ for bisdemethoxycurcumin, demethoxycurcumin, and curcumin, respectively) and high loading capacity of curcuminoids.

- The interaction between curcuminoids and phospholipid by a hydrogen bond was confirmed by differential scanning calorimetry (DSC), powder X-ray diffraction (XRD), fourier transform infrared (FT-IR), and ${ }^{1} \mathrm{H}$ nuclear magnetic resonance ( $\left.{ }^{1} \mathrm{H}-\mathrm{NMR}\right)$.

- Curcuminoids-loaded nano phytosomes showed significantly higher cellular uptake and in vitro cytotoxicity profile on breast cancer cell line compared to raw curcuminoids indicating the potential of phytosomal nanoparticles as a promising delivery system for natural herbal phytoconstituents for anticancer applications.

\section{INTRODUCTION}

Curcuminoids are major active ingredients extracted from turmeric (Curcuma longa) including three phenolic components: curcumin, DMC, and BDMC which was widely used as a spice and a traditional medicine thousands of years ago in Southeast Asia, China and India. ${ }^{1}$ Till date, many studies have been focused on evaluation of the chemical, physical and biological activity of curcumin, the most abundant substance in curcuminoids. Accumulating evidence has shown that curcumin has antioxidant, antiinflammatory, anti-microbial, anti-parasitic, antimutagen and anticancer properties. ${ }^{2}$ Additionally, recent studies also demonstrated that DMC and $\mathrm{BDMC}$ also has been promising biological activities, including anticancer effect. ${ }^{2-5}$ Notably, remote curcumin has been shown to be less potent than the whole curcuminoids in anti-inflammatory, ${ }^{6,7}$ antioxidant, ${ }^{8}$ and anticancer effect. ${ }^{4}$ Thus, in several cases, treatment with whole curcuminoids may provide more therapeutic benefits than those received single curcumin.

Breast cancer has been considered as a major public health problem worldwide in women. ${ }^{9}$ Current medications and treatments including surgery, radiotherapy, chemotherapy, and/or hormone therapies are useful for treating breast cancer. The 
development of natural drugs delivery systems with low toxicity and high selectivity compared to synthetic compounds are essential for chemotherapy treatments. Curcuminoids have been considered as a natural anti-breast cancer agents. The effects of curcuminoids have been studied in several cancer cell lines including breast cancer. Curcuminoids were found to be potential cytotoxic agents, which could be promising compounds against selective cancer. ${ }^{10,11}$

Due to poor water solubility, low absorption from the gut, fast metabolism, and fast systemic elimination of curcuminoids, some formulations have been applied to improve the bioavailability of curcuminoids including the nanostructured lipid carrier system, ${ }^{12}$ curcuminoids- lecithin formulation. ${ }^{13}$ Among these nanoplatforms, nanosized phytosomes have been reported to offer many advantages in the enhancement of therapeutic efficacy of curcuminoids. Remarkably, after oral administration, phyto-phospholipid complex possibly entered into the intestine by direct solubilization or endocytosis, ${ }^{14}$ by paracellular transport in lateral tight junctions ${ }^{15}$ or lymphatic port. ${ }^{16-}$ ${ }^{18}$ The major benefit of lymphatic transport is to bypass the first-pass metabolism and applicable for targeted drug delivery. ${ }^{14,19}$ It is reasonable to prepare a formulation with curcuminoids loaded in nano phytosome to enhance anticancer activites of curcuminoids.

Therefore, in this study, we utilized the evaporation method combined the extrusion technique to prepare phytosomal curcuminoids nanoparticles (Curs-Phyto). The physicochemical properties of curcuminoids-loaded nano-phytosome were confirmed by dynamic light scattering (DLS), transmission electron microscopy (TEM), differential scanning calorimetry (DSC), powder X-ray diffraction (XRD), fourier transform infrared (FT-IR), and ${ }^{1} \mathrm{H}$ nuclear magnetic resonance ( $\left.{ }^{1} \mathrm{H}-\mathrm{NMR}\right)$. Furthermore, to investigate the compatibility of Curs-Phyto for oral delivery, their stability after lyophilization and drug release profiles in simulated gastric and intestinal fluids were also evaluated. We hypothesized that the phytosomal formulation could significantly improve cellular uptake of curcuminoids on MCF-7 cancer cells, resulting in promoting the cytotoxicity towards this cancer cell line.

\section{MATERIALS AND METHODS}

\section{Materials}

Curcuminoids (curcumin 79\%, demethoxycurcumin $17 \%$ and bisdemethoxycurcumin 3.7\%) was obtained from Laurus Labs Private Limited, JN Pharma City (India). HEPC (L- $\alpha$-phosphatidylcholine, hydrogenated) was purchased from Avanti Polar Lipids (USA). Cholesterol was procured from Sigma-Aldrich Corp (St. Louis, MO, USA). Dulbecco's modified Eagle's medium (DMEM), fetal bovine serum (FBS), penicillin/streptomycin was provided from GE Healthcare Life Sciences (Marlborough, MA, USA).

\section{Cell line}

The MCF-7 breast cancer cell line was purchased from the Korean Cell Line Bank (KCLB) (Seoul, South Korea). The cells were cultivated in a humidified incubator (Thermo Fisher Scientific, USA) at $37^{\circ} \mathrm{C}$ in an atmosphere of $5 \% \mathrm{CO}_{2}$. The cells were cultured in DMEM supplemented with $10 \%(\mathrm{v} / \mathrm{v}) \mathrm{FBS}$, and $1 \%(\mathrm{v} / \mathrm{v})$ penicillin/streptomycin solution.

\section{Preparation curs-phyto}

The Curs-Phyto comprising Curcuminoids, HEPC, cholesterol with molar ratio 1:2:0.1 was prepared by evaporation method. Briefly, Curcuminoids, HEPC and cholesterol was dissolved in ethanol. The mixture was poured in a round bottom flask and the reaction was performed in a rotary evaporator (EYELA, USA) at room temperature. After $2 \mathrm{~h}$, all the organic solvent was removed by rotary evaporation in vacuum until the thin film was produced in the round bottom flask. Thereafter, the film was hydrated with distilled water at $40^{\circ} \mathrm{C}$. Free drugs were eliminated using ultra filtration tube (MWCO: 30 $\mathrm{kDa}$ ). Then, the resultant phytosomes were extruded through a 0.2 $\mu \mathrm{m}$ membrane, followed by lyophilized and kept in $-20^{\circ} \mathrm{C}$ for further evaluation. Blank phytosomes were prepared under the same condition as above description except for the addition of curcuminoids.

\section{Physicochemical characterization of curs-phyto}

\section{Determination the complexation rate and drug loading capacity}

The complexation rate (\%) of Curs-Phyto calculated separately for Cur, DMC and BDMC were determined by HPLC-UV methods. The mobile phase consisted of ACN ( $0.04 \%$ acetic acid, v/v) and formic acid $0.01 \%$ at a volume ratio of $60: 40 . \mathrm{C}_{18}$ column $\left(\mathrm{RP} \mathrm{C}_{18}, 4.6 \times 150 \mathrm{~mm}, 5 \mu \mathrm{m}\right)$ was used as the stationary phase. The flow rate of mobile phase was 1 $\mathrm{mL} / \mathrm{min}$; injection volume was $5 \mu \mathrm{l}$ and the column temperature was controlled at $25^{\circ} \mathrm{C}$. A wavelength of $430 \mathrm{~nm}$ was used for detection. The complexation rate (CR\%) and drug loading capacity (DC\%) of Cur, $\mathrm{DMC}$ and $\mathrm{BDMC}$ were calculated according to the equations described below

$\mathrm{CR} \%=\left(\mathrm{W}_{\text {total }}-\mathrm{W}_{\text {free }}\right) / \mathrm{W}_{\text {total }} \times 100$

Where $\mathrm{W}_{\text {free }}$ is the amount of free drug measured in the lower chamber of Millipore Amicon ${ }^{\otimes}$ Ultra filtration tube after centrifugation and $\mathrm{W}_{\text {total }}$ is the initial amount of drug

$\mathrm{DC} \%=\mathrm{W}_{\text {complexed }} / \mathrm{W}_{\text {phytosome }} \times 100$

Where $\mathrm{W}_{\text {complexed }}$ is the amount of the complexed curcuminoids and $\mathrm{W}_{\text {phytosome }}$ is the weight of phytosome curcuminoids.

\section{Particle size, size distribution and zeta-potential}

Phytosomal suspensions were diluted with purified water. The Z-averaged hydrodynamic particle sizes, polydispersity indexes (PDI), and $\zeta$-potential of Curs-Phyto were measured by the Zetasizer Nano ZS (Malvern Instruments, Malvern, UK).

\section{Morphological characterization}

The morphology of Curs-Phyto was confirmed by a transmission electron microscope (TEM, CM 200 UT, Philips, MA, USA), with 2\% $\mathrm{w} / \mathrm{v}$ phosphotungstic acid prestaining treatment.

\section{Differential scanning calorimetry (DSC)}

A differential scanning calorimeter (DSC Q200, TA Instruments) was used to measure enthalpy and melting point of all substances used in this study. Samples were heated in the range of $0-200^{\circ} \mathrm{C}$ at a scanning rate of $20^{\circ} \mathrm{C} / \mathrm{min}$.

\section{Powder X-ray diffraction (XRD)}

The X-ray diffraction (XRD) analysis of Cur-Phyto, curcuminoids, HEPC, and cholesterol were measured by X'Pert PRO diffractometer (PAN analytical, Almelo, The Netherlands), which used monochromatic, $\mathrm{Ni}$-filtered $\mathrm{Cu} \mathrm{Ka}$-radiation $\left(40 \mathrm{kV}\right.$ voltage, scanning rate $=0.013^{\circ}$ min -1 ) over diffraction angle $(2 \theta)=10^{\circ}-60^{\circ}$.

\section{Fourier transform infrared (FTIR)}

Spectroscopic analysis Curs-Phyto, curcuminoids, HEPC, and cholesterol were analyzed by FTIR spectroscopy using Thermo Scientific Nicolet Nexus 670 FTIR spectrometer with Smart iTR and diamond window (Thermo Fisher Scientific Inc., Waltham, MA, USA). The spectra were recorded from 600 to $4000 \mathrm{~cm}^{-1}$.

\section{$1 H-N M R$}

The ${ }^{1} \mathrm{H}$ nuclear magnetic resonance (500 $\mathrm{MH}_{\mathrm{Z}} \mathrm{CDCl}_{3}, \delta$ in ppm) spectroscopy of curcuminoids, HEPC, cholesterol and Curs-Phyto 
were carried out by Brucker Advance DRX-500, BruckerBioSciences Corporation, Billerica, MA, USA) at $500 \mathrm{MHz}$.

\section{In vitro drug release}

The in vitro drug release was studied in $\mathrm{pH} 1.2$ and $\mathrm{pH} 6.8$ buffers to simulate almost all the physiological conditions encountered during the journey of a delivery system following oral administration. Briefly, Curs-Phyto was incubated at $37^{\circ} \mathrm{C}$ with continuous shaking $100 \mathrm{rpm}$ up to $2 \mathrm{~h}$ (in the case of $\mathrm{pH} 1.2$ ) or up to $6 \mathrm{~h}$ (in the case of $\mathrm{pH} 6.8$ ) in separate centrifuge tubes. The above time intervals were selected for the study based on the expected residence time in the stomach and intestine. At predetermined intervals of time, released amount of Cur, DMC and BDMC in Curs-Phyto was quantified by HPLC-UV method with analytical conditions described above. ${ }^{17}$

\section{In vitro cytotoxicity assay}

In vitro cytotoxicity of blank phytosome, free curcuminoids and CursPhyto on MCF-7 cell line were carried out by MTS cell viability assay. Briefly, MCF-7 cells were cultured in a 96-well plate at a density of $1 \times 10^{4}$ cells/well. After $24 \mathrm{~h}$, the cells were treated with blank phytosome, free curcuminoids and Curs-Phyto at the range of concentrations: 0.1, 1, 5, $10,20,50 \mu \mathrm{g} / \mathrm{mL}$ and incubated for $24 \mathrm{~h}$. To finalize, MTS treatment was performed, following the manufacturer's instructions, and the absorbance at $493 \mathrm{~nm}$ was measured using an automated microplate reader (Thermo Fisher Scientific, USA). Untreated cells were used as controls. The percentage viability was calculated as:

Cell viability $(\%)=\left(\mathrm{A}_{93(\text { treated group })}-\mathrm{A}_{0}\right) /\left(\mathrm{A}_{493(\text { without treatment) }}-\mathrm{A}_{0}\right) \times 100$

A493 (treated group) refers to the groups of cells treated with blank phytosome, free curcuminoids and Curs-Phyto at different concentrations.

A493 (without treatment) refers to the control group of cells without any drug treatment, while A0 refers to medium only.

\section{Cell cycle analysis}

Cell cycle arrest caused by the free curcuminoids and Curs-Phyto was determined using the Cell-Clock ${ }^{\mathrm{mix}}$ Assay kit (Biocolor Ltd., UK), which can distinguish the four major phases (G1, S, G2, and M) of the mammalian cell cycle. Initially, MCF-7 cells were seeded at a density of $1 \times 10^{5}$ cells per well in a 12 -well plate and incubated for $24 \mathrm{~h}$. These cells were then treated with free curcuminoids or Curs-Phyto and incubated for $24 \mathrm{~h}$. Finally, the treated cells were stained with the supplied redox dye (Cell-Clock Dye Reagent) for $1 \mathrm{~h}$ at $37^{\circ} \mathrm{C}$ and images were captured with a fluorescence microscope (Nikon Eclipse Ti, Nikon Instruments Inc.). The percentage of cells arrested in each phase was obtained from digitized photomicrographs using ImageJ software.

\section{Cellular uptake studies}

Cellular uptake of curcuminoids from free curcuminoids and curcuminoids-loaded nano-phytosomes were determined by fluorescence microscopy and fluorescence-activated cell sorting (FACS) analysis based on the advantage of the intrinsic red fluorescence of curcuminoids. ${ }^{20}$

The internalization of Curs-Phyto and free curcuminoids by breast cancer cells were conducted using confocal laser scanning microscopy (K1-Fluo CLSM, Nanoscope Systems Inc, Korea). Briefly, MCF-7 cells were seeded on coverslips placed in 12 -well plates at a density of $1 \times 10^{5}$ cells $/ \mathrm{mL}$ and were incubated for $24 \mathrm{~h}$ for cell attachment. The cells were incubated with the free curcuminoids or Curs-Phyto at the equivalent concentration of curcuminoids for the pre-designed time period. After removing the media, the coverslips were gently washed with PBS, fixed with $4 \%$ paraformaldehyde solution in the dark and stained with 4',6-diamidino-2-phenylindole (DAPI).
The uptake of Curs-Phyto was studied using fluorescence-activated cell sorting (FACS; BD Biosciences, San Jose, CA, USA). MCF-7 cells (2 $\times 10^{5}$ / well), were seeded in 6 wells- plates and incubated for $24 \mathrm{~h}$. In order to evaluate concentration-dependent or time-dependent uptake profiles of Curs-Phyto on MCF-7 cells, cells were treated with this formulation at different times or concentrations. After treatment, the cells were collected, washed three times with PBS, and re-suspended in $1 \mathrm{~mL}$ PBS for FACS analysis.

\section{RESULTS AND DISCUSSION}

\section{Preparation and characterization of curs-phyto}

Curs-Phyto was prepared by evaporation methods combined extrusion technique with a molar ratio of Curcuminoids: HEPC: Cholesterol = 1:2:0.1. The images of curcuminoids, HEPC, Cholesterol, their physical mixture, and Curs-Phyto were shown in Figure 1.

\section{Physicochemical characterization of curs-phyto}

The particle size, size distribution, and zeta potential of Curs-Phyto are essential due to its effects on the physicochemical stability and cellular uptake of the drug delivery system for phytoconstituents. Smaller particles generate a larger surface area and have faster absorption. ${ }^{21}$ The nanoscale particle size with a narrow particle size distribution is useful for cancer therapy via enhanced penetration and retention (EPR) effect, ensuring the passive tumor targeting mechanism. ${ }^{22,23}$ Not only did the Z-average particles size of Curs-Phyto is smaller than $200 \mathrm{~nm}$ but the PDI of Curs-Phyto also was lower than 0.2 which indicated better distribution. The zeta potential of Curs-Phyto was approximate -6.19 $\mathrm{mV}$ (Figure $2 \mathrm{~B}$ ), which is facilitated for the stability of nanoparticles as well as reducing the nonspecific interactions with negatively charged red blood cells and serum proteins. ${ }^{24}$ It is well-known that if nanoparticles were in the aqueous suspension, the nano systems would be destroyed on account of oxidation of phospholipid, curcuminoids leakage, particle gathering and/or the biological interference. ${ }^{18}$ In this study, the lyophilization method was utilized to maintain the long-term physicochemical stability of the nanoparticle system. After reconstruction in distilled water, the average particle size became marginally larger, with PDI also moderately higher compared to that of the Curs-Phyto before lyophilization, possibly attributed to a partial aggregation between particles. However, there was no considerable change in zeta potential before and after lyophilization (Figure 2D). Thus, the lyophilization technique served as an effective technique to remain the physicochemical stability of Curs-Phyto.

The morphology of Curs-Phyto was observed by TEM and the image (Figure 2C) showed curcuminoids-loaded nano phytosomes were

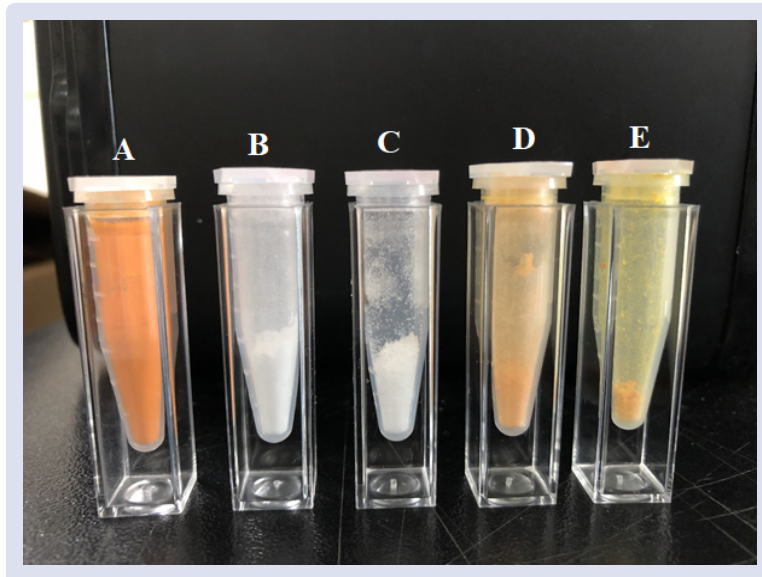

Figure 1: The images of A) Curcuminoids; B) HEPC; C) Cholesterol; D) Physical mixture; E) Curs-Phyto. 
regularly spherical in shape and the particle size was consistent with that determined by DLS.

The complexation rate (CR\%) of Cur, DMC and BDMC and drugloading capacity (DC\%) of Curs-Phyto were determined by HPLC-UV analysis. An HPLC chromatographical of Curs-Phyto was shown in Figure 3.

Table 1 indicated that the complexation rate of BDMC, DMC, and Cur in Curs-Phyto were approximately $87.94 ; 95.20$ and $90.23 \%$, respectively. The Cur-loading capacity of Curs-Phyto was $12.45 \%$ which is the highest in comparison to that of DMC or BDMC-loading capacity. When considering as a whole curcuminoids-loading capacity of Curs-Phyto was determined as high as $16.55 \%$. This may be ascribed to the interaction between phytoconstituents and phospholipid. After complexing with Cur, DMC, BDMC, the polar head of HEPC was connected to the phenolic hydroxyl groups of Cur and its derivatives by a hydrogen bond. This bond was confirmed by DSC, FTIR, XRD and ${ }^{1} \mathrm{H}-\mathrm{NMR}$ analysis below.

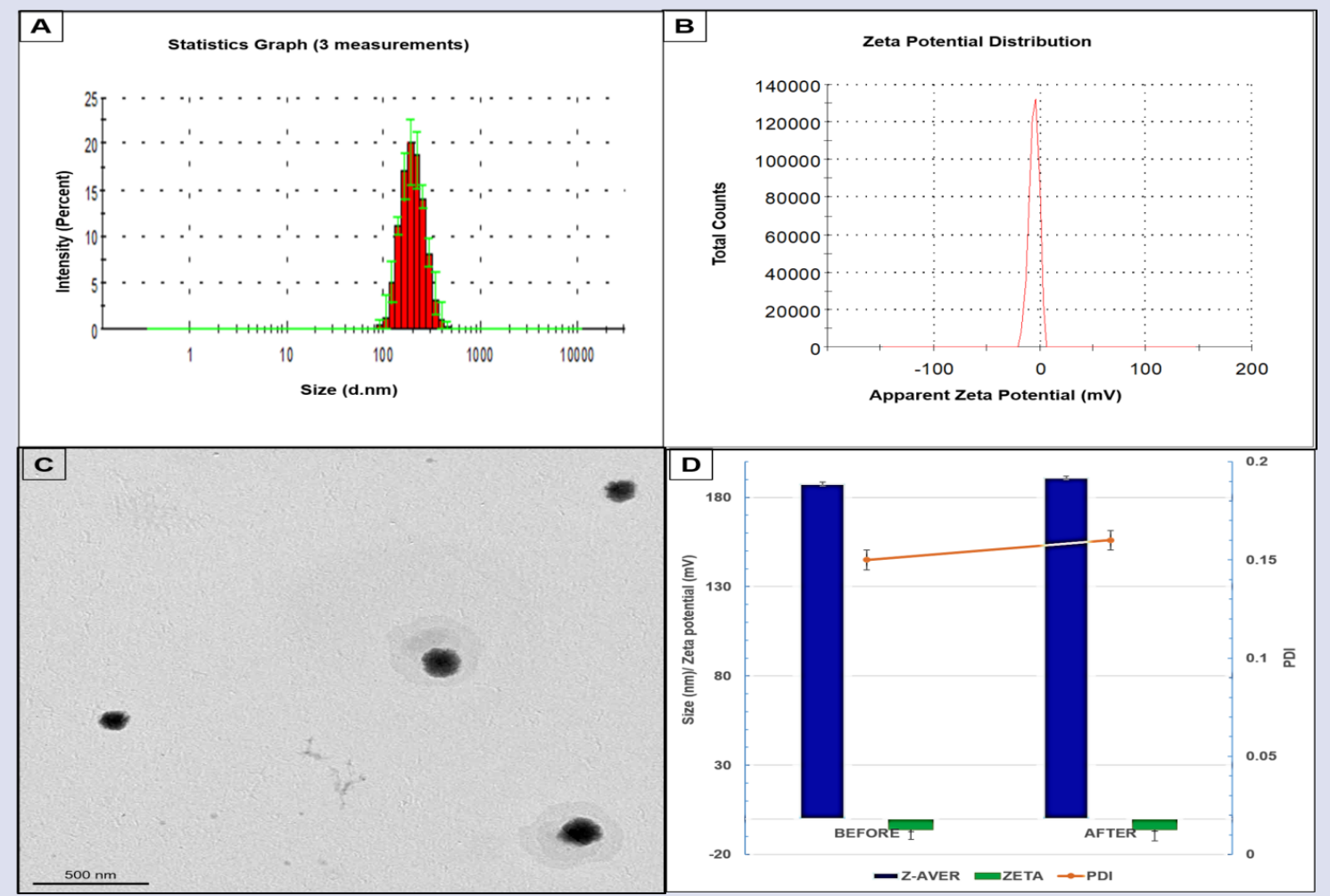

Figure 2: A) Particle size and B) Zeta potential of Curs-Phyto determined by the dynamic light scattering (DLS); C) TEM image of Curs-Phyto; D) The particles size, PDI and Zeta potential of Curs-Phyto before and after lyophilization.

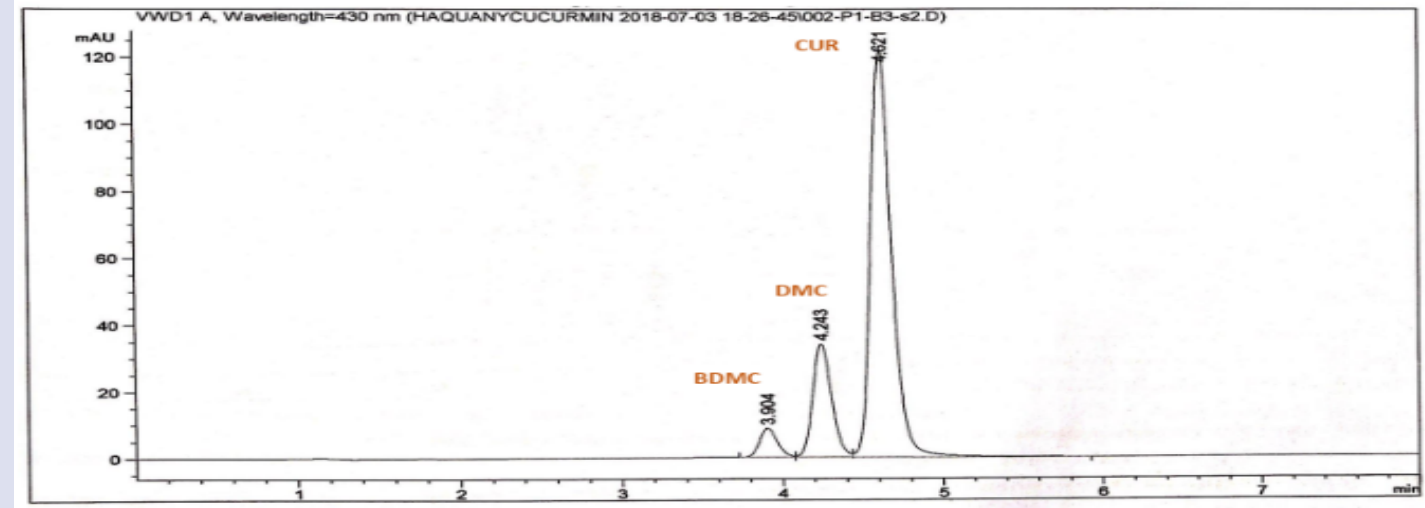

Figure 3: Representative HPLC chromatograms of BDMC, DMC and CUR.

Table 1: The complexation rate (\%) and drug-loading capacity (\%) of curs-phyto calculated separately for Cur, DMC and BDMC.

\begin{tabular}{ccc}
\hline & Complexation rate (CR \%) & Drug-loading Capacity (DC \%) \\
\hline BDMC & $87.94 \pm 3.32$ & $0.67 \pm 0.02$ \\
DMC & $95.20 \pm 2.01$ & $3.42 \pm 0.04$ \\
CUR & $90.23 \pm 1.41$ & $12.45 \pm 0.39$ \\
\hline
\end{tabular}




\section{Differential scanning calorimetry (DSC)}

The DSC thermographs of curcuminoids, HEPC, cholesterol, and CursPhyto were illustrated in Figure 4A. Thermogram of curcuminoids showed a single peak with an onset of $170.53^{\circ} \mathrm{C}$ and maximum occurrence at $178.51^{\circ} \mathrm{C}$ corresponding to its melting point. DSC thermograms of HEPC and cholesterol also illustrated endothermic peaks at $72.05^{\circ} \mathrm{C}$ and $149.90^{\circ} \mathrm{C}$, respectively. One broad peak at $95.38^{\circ} \mathrm{C}$ appeared in the DSC thermogram of Curs-Phyto, which is distinct from the peaks of the individual components in phytosomal formulation. The disappearance of the melting peak of curcuminoids and remarkable shift of the melting peak of cholesterol to the lower melting points in Curs-Phyto thermogram indicated that curcuminoids were distributed on the surface and inside the matrix of nano phytosome and lost its crystalline structure.

That curcuminoids and HEPC interacted by hydrogen bonding between $-\mathrm{OH}$ group of phenol rings of curcuminoids and hydrophilic region of HEPC led to the long hydrocarbon tail of HEPC to turn freely and envelop the hydrophilic region of phospholipids containing the curcuminoids molecules. The decline in the melting point of cholesterol in phytosome compared with the pure cholesterol could be attributed to its incorporation into the bilayer of phytosome leading to the more ordered structure. ${ }^{25}$

\section{Powder X-ray diffraction (XRD}

The XRD spectra of curcuminoids, HEPC, cholesterol, and Curs-Phyto were showed in Figure 4B. Both sharp crystalline peaks and broad peaks which were exhibited in the XRD spectra of curcuminoids, HEPC and cholesterol indicated a crystalline form of these components. Notwithstanding, the crystalline signals disappeared in the XRD spectrum of Curs-Phyto. The results further revealed that curcuminoids uniformLy dispersed in the HEPC-cholesterol matrix.

\section{Fourier transform infrared (FTIR)}

FTIR spectra of curcuminoids, HPEC, cholesterol, and Curs-Phyto were illustrated in the Figure 4C. FTIR spectrum of curcuminoids exhibited the IR band at $3356 \mathrm{~cm}^{-1}$ due to $\mathrm{O}-\mathrm{H}$ stretching vibration with a wide absorption peak $\left(3354 \mathrm{~cm}^{-1}\right)$ of HEPC shifted to a lower wave number at $3330 \mathrm{~cm}^{-1}$ of Curs-Phyto to form a wider absorption peak. Additionally, a shift of the $\mathrm{PO}_{4}$ stretching vibration at $1234 \mathrm{~cm}^{-1}$ in the
FT-IR spectrum of the HEPC to a lower wave number at $1219 \mathrm{~cm}^{-1}$ in that of phytosomal formulation indicated that the phenol hydroxyl group of curcuminoids interacted with the phosphate group of HEPC by hydrogen bonding in the structure of Curs-Phyto.

The $-\mathrm{CH}_{2}$ asymmetric stretching mode near $2916 \mathrm{~cm}^{-1}$ and $2849 \mathrm{~cm}^{-1}$ were generally the strongest bands in the spectrum of HEPC. It is well-known that the frequencies of the $\mathrm{CH}_{2}$ stretching modes depend on the chain confromational disorder of the long-chain fatty acids. A shift to lower frequency could be further explained by a more ordered structure in phytosomal curcuminoids. ${ }^{26,27}$ The IR spectra showed that cholesterol increases the head group hydration and decrease inter chains order within the bilayer. That insertion of cholesterol into the membranes of phytosome led to a shift to a lower frequency in the $\mathrm{CH}$ bands and a lower frequency and broadening of the $\mathrm{PO}_{2}-$ asymmetric stretching mode for the spectrum of phytosome indicated a more ordered structure in the hydrophobic head of HEPC. This finding is consistent with other studies. ${ }^{28,29}$

\section{${ }^{1} \mathrm{H}-\mathrm{NMR}$}

Figure 5 showed the ${ }^{1} \mathrm{H}-\mathrm{NMR}$ spectra of curcuminoids, HEPC, Cholesterol, and Curs-Phyto. Curcuminoids: ${ }^{1} \mathrm{H}-\mathrm{NMR}$ (d ppm): 7.60 (dd, 2H, H-4, 4'), 7.12 (dd, 2H, H-6, 6’), 7.05 (d, 2H, H-10, 10'), 6.92 (d, $\left.2 \mathrm{H}, \mathrm{H}-9,9^{\prime}\right), 6.48\left(\mathrm{dd}, 2 \mathrm{H}, \mathrm{H}-3,3^{\prime}\right), 5.86(\mathrm{~s}, 1 \mathrm{H}, \mathrm{H}-1)$. That the signal of protons at $\delta 0.889$ and $2.338 \mathrm{ppm}$ was due to signals of protons of methyl and methylene protons linked to $-\mathrm{C}(=\mathrm{O})$ - $\mathrm{C}$ group in ${ }^{1} \mathrm{H}-\mathrm{NMR}$ of Curs-Phyto were also characteristics of the non polar head of HEPC. The signal at $\delta 3.379 \mathrm{ppm}$ was due to protons of a methyl group attached to $\mathrm{N}$-atom of choline which showed a signal spreading due to their connection in Curs-Phyto. This proton exchange occurred rapidly and caused a wide amplitude of the peak, which weakened the NMR signals of these groups. Thus, by the ${ }^{1} \mathrm{H}-\mathrm{NMR}$ spectrum, the linker between a phenyl group of curcuminoids and choline part of HEPC was confirmed.

Taken together, these results proved the presence of hydrogen bonds interaction between curcuminoids and polar head of HEPC coupled with insertion of cholesterol into the membranes of Curs-Phyto which led to the physicochemical stability of phytosomal nanoparticles. ${ }^{25,30}$ This might be facilitated for the enhancement in cellular uptake of Curs-Phyto via oral administration.

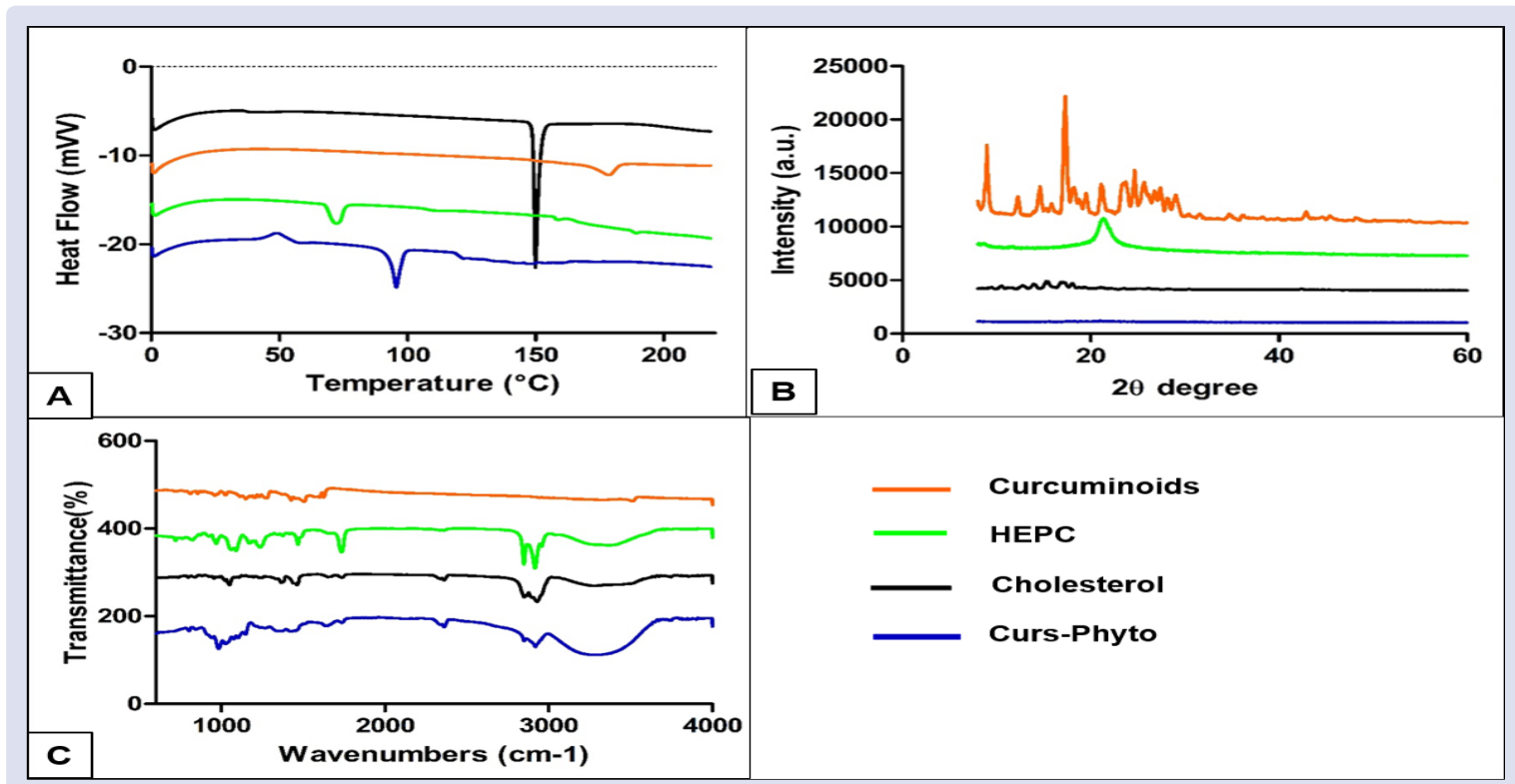

Figure 4: A) Differential scanning calorimetry (DSC) thermogram; B) X-ray Diffraction (XRD) diffractograms; C) Fourier Transform Infrared (FTIR) of Curcuminoids, HEPC, Cholesterol, and Curs-Phyto. 


\section{In vitro drug release profiles}

Figure 6 showed the in vitro drug release profiles of Curs-Phyto separately calculated for Cur, DMC and BDMC. In the in vitro release studies, artificial gastric fluid $(0.1 \mathrm{M} \mathrm{HCl}$ with $\mathrm{pH} 1.2)$ and artificial intestinal fluid (PBS with $\mathrm{pH}$ 6.8) were chosen as the dissolution media for Curs-Phyto to imitate its oral administration environment. It was clear that the rate of Cur, DMC and BDMC release in the artificial intestinal fluid were higher than that in artificial gastric fluid. It was explained that phytosomal particles might disintegrate faster in the neutral medium, and curcuminoids molecules would be more easily ionized in intestinal juice with higher $\mathrm{pH}$ values, hence facilitating the escape of entrapped drug from nanoparticles. After $2 \mathrm{~h}$ incubated in $\mathrm{pH}$ 1.2 buffer, $54.87 \%, 82.72 \%$ and $92.6 \%$ of the amount of BDMC, DMC, and CUR, respectively, were associated with Curs-Phyto. The respective values in $\mathrm{pH} 6.8$ buffer after $6 \mathrm{~h}$ incubation were 48.68\%, 75.51\%, $85.35 \%$ of BDMC, DMC and CUR, respectively. This form would be available for its absorption via enterocytes. Compared with two derivatives, CUR appears to be more stable in the Curs-Phyto in both testing environments. This may be ascribed to the chemical structure distinction of three components extracted from turmeric. ${ }^{18}$

\section{In vitro cytotoxicity}

The effects were observed in a concentration-dependent manner as concentration increased from 0.1 to $50 \mu \mathrm{g} / \mathrm{mL}$. After incubating MCF7 cells with blank phytosome, free curcuminoids, and Curs-Phyto at concentrations range of $0.1,50 \mu \mathrm{g} / \mathrm{mL}$, cell cytotoxicity of these formulations were determined by MTS assay. As shown in Figure 7A, 7B in comparison with free curcuminoids, Curs-Phyto showed remarkably enhanced cytotoxicity toward MCF-7 cells. There was a decrease in the proportion of cell viability caused by an increasing curcuminoids concentration tendency. More importantly, it was found that blank phytosome had no significant cytotoxicity against MCF-7 cells, even at the concentration of $50 \mu \mathrm{g} / \mathrm{mL}$. Thus, the cytotoxicity of the Curs-Phyto was induced by the released curcuminoids and not by the toxic effect of HEPC and cholesterol. It was noticed that the CursPhyto generated significantly greater cytotoxicity compared with free drug. For MCF-7 cells, the half-maximal inhibitory concentration (IC50) value of free curcuminoids and Curs-Phyto were determined as 20.2 and $11.6 \mu \mathrm{g} / \mathrm{mL}$, respectively, for $24 \mathrm{~h}$ of incubation.
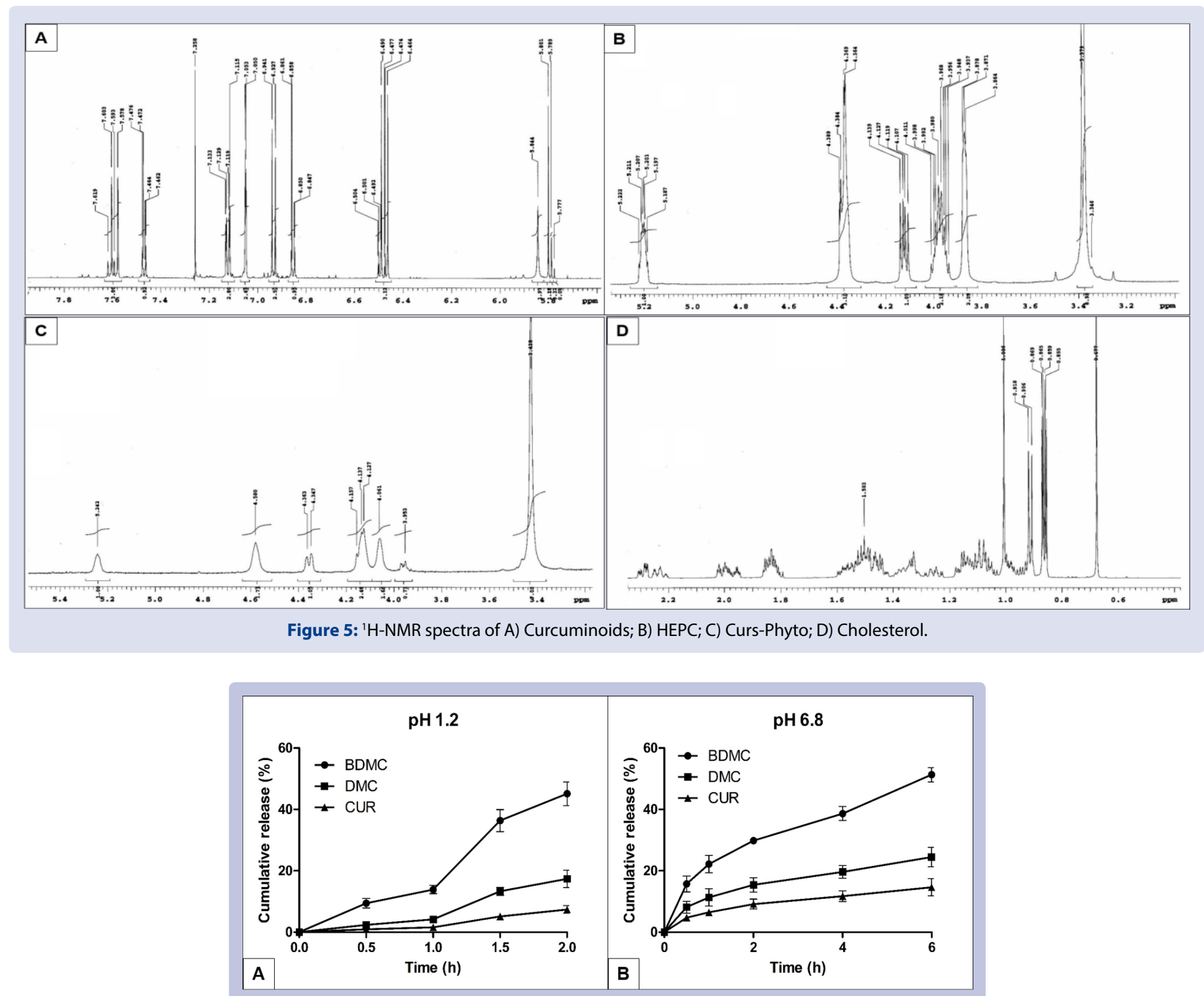

Figure 6: In vitro time-dependent drug release profiles of CUR, DMC and BDMC from the phytosomes in $\mathrm{pH} 1.2$ (A) and pH 6.8 (B) buffer. 


\section{Cell cycle analysis}

In order to study cell cycle of MCF-7 cells following treatment with free curcuminoids and Curs-Phyto, the Cell-Clock ${ }^{\mathrm{ma}}$ Assay kit was utilized with a redox dye, which changed color according to the cell cycle phases (G0/G1, S, G2/M). The percentages of cells in their respective phases were shown in Figure 7C, 7D both free curcuminoids and Curs-Phyto at an equivalent dose of $10 \mu \mathrm{g} / \mathrm{mL}$ significantly altered the distribution of MCF-7 cells in the cell cycle phases. In comparison to untreated cells, curcuminoids and Curs-Phyto increased the portion of cells in the G0/ G1 phase by $67.55 \%$ and $70.06 \%$, respectively. Concomitant with this augment was a decline in the proportion of G2/M-phase cells by $7 \%$ and $68 \%$. Similarly, the descent of $8.31 \%$ for cells treated with the free drug and $10.61 \%$ for those with formulation were recorded in S phase. Curcuminoids, in both free form and formulated Curs-Phyto, induced accumulation of MCF-7 cells in the G0/G1 phase of the cell cycle. It is clear that curcuminoids could inhibit cellular proliferation in MCF-7 cells in vitro. These results are consistent with that of Hua-Qiang Li et al., who reported that curcumin arrested the cell cycle at G1 phase of MCF-7 cells. $^{31,32}$

\section{Cellular uptake efficacy of curs-phyto}

We investigated the cellular uptake of free curcuminoids and CursPhyto by MCF-7 cells using laser confocal scanning microscopy and flow cytometry. MCF-7 cells were incubated with free curcuminoids and its Curs-Phyto formulation at $37^{\circ} \mathrm{C}$ for 30 mins. The greater red fluorescence intensity was clearly observed in MCF-7 cells treated with
Curs-Phyto in comparison to that treated with free drugs (Figure 8D). This result was well consistent with the quantitative result determined by flow cytometry. The data from flow cytometry in Figure $8 \mathrm{C}$ indicated that Curs-Phyto had significantly higher uptake than free curcuminoids. MCF-7 cells treated with Curs-Phyto showed a prominent right shift upon cytometric analysis, indicating a greater cellular uptake than free curcuminoids. This may be attributed to particle size and structure characteristics of phytosomal formulation Curs-Phyto. The smaller size of the particle, the easier it can be taken up by the cells. The similar structure component and high affinity between the phospholipid on the phytosome Curs-Phyto's surface phospholipid and cell membranes are crucial determinants. ${ }^{30}$ Phytosome can easily move from a hydrophilic environment into the lipophilic environment of the cell membrane and then enter the cell. ${ }^{33}$ Our data demonstrated that Curs-Phyto exhibited higher association and internalization into the cells compared to that of free curcuminoids, which proved that the phytosomal formulation has a profound impact on the uptake of curcuminoids. Curcuminoids were taken up more efficiently when formulated in phytosome.

Moreover, qualitative time- and concentration -dependent uptake profile of Curs-Phyto were examined using FACS analysis. (Figure $8 \mathrm{~A}, 8 \mathrm{~B})$. The percentage of gated fluorescing cells analyzed by flow cytometry increased as the concentration of curcuminoids added into the cell culture increased ( 1 to $5 \mu \mathrm{g} / \mathrm{mL}$ ), which showed a concentrationdependent increase. However, there was no significant change in fluorescence intensity in MCF-7 breast cancer cell line although treatment time was increased (from 30 to 90 mins). Thus, the cellular uptake of Curs-Phyto was completely time-independent.

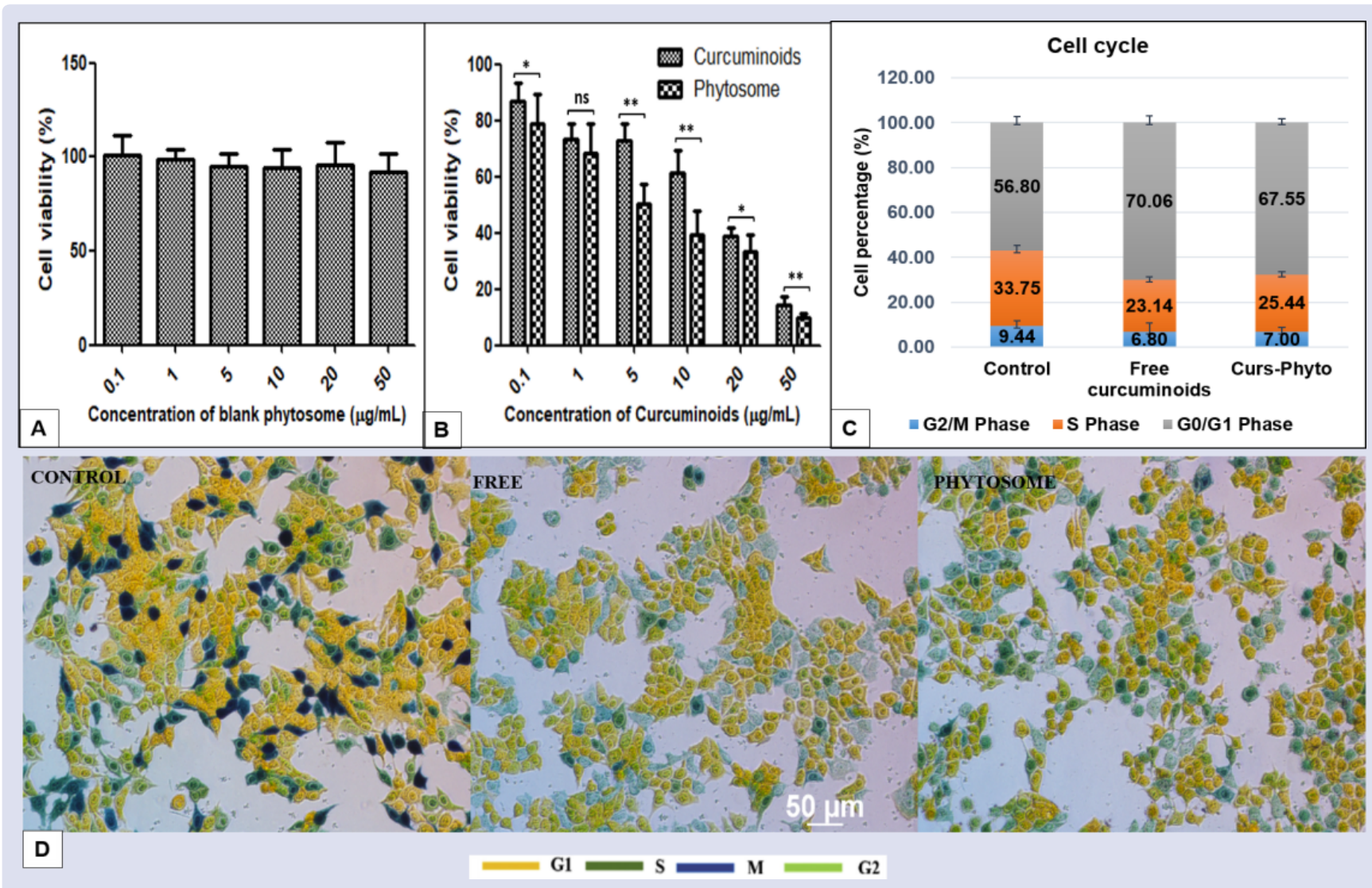

Figure 7: In vitro cytotoxicity on cancer cells. MTS assay evaluated the cell viability of MCF-7 cells after incubation with (A) blank phytosome, (B) free curcuminoids and Curs-Phyto for 24h. (C) quantification of percentage of MCF-7 cells in the respective phases and (D) Cell cycle analysis of MCF-7 cells treated with free curcuminoids and Curs-Phyto (Scale bars $=50 \mu \mathrm{m}$ ). Data are represented as mean $\pm S D, " p<0.05$, " $p<0.001$, ns: not significant. 


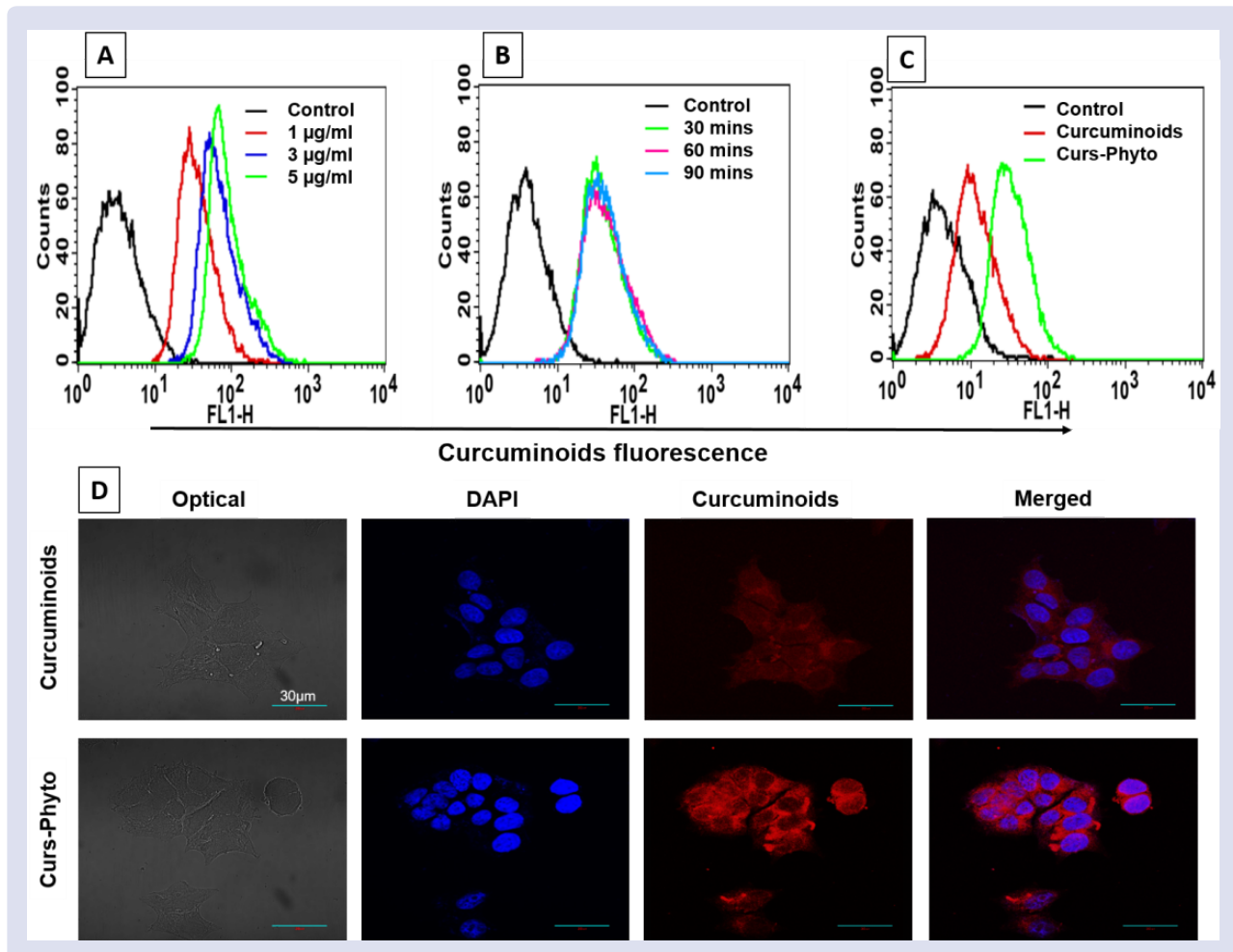

Figure 8: Cellular uptake investigation. Flow cytometric analysis of Curs-Phyto uptake into MCF-7 cells in (A) concentration-dependent manner, (B) time-denpendent manner, and (C) the uptake free curcuminoids and Curs-Phyto in MCF-7 cells after incubation for 30 minutes. (D) Confocal microscopy images of MCF-7 cells after incubation with free curcuminoids and Curs-Phyto for 30 minutes.

\section{CONCLUSION}

Curcuminoids-loaded nano-phytosomes were successfully prepared by the evaporation combined extrusion technique. Curs-Phyto exhibited a nanometer range in particle size and a spherical structure in shape. Not only were Cur, DMC and DMC loaded in phytosomal nanoparticles with high complexation rate and drug-loading capacity, but this formulation also showed better performance of in vitro cytotoxicity and cellular uptake on MCF-7 cells compared to free drugs. Our experimental results may suggest the potential of phytosomal nanoparticles to be considered as a promising delivery system for natural herbal phytoconstituents for anticancer applications.

\section{ACKNOWLEDGMENTS}

This research was supported by the Project on Science and Technology from Vietnam Ministry of Science and Technology on Applied Research and Advanced Technology Development for Protection and Care of the People's Health (Code KC.10.12/16-20).

\section{CONFLICTS OF INTEREST}

The authors declare no conflict of interest.

\section{REFERENCES}

1. Goel A, Kunnumakkara AB, Aggarwal BB. Curcumin as "Curecumin": From kitchen to clinic. Biochem Pharmacol. 2008;75(4):787-809.

2. Ahsan H, Parveen N, Khan NU, Hadi SM. Pro-oxidant, anti-oxidant and cleavage activities on DNA of curcumin and its derivatives demethoxycurcumin and bisdemethoxycurcumin. Chem Biol Interact. 1999;121(2):161-75.

3. Kim DS, Park SY, Kim JK. Curcuminoids from Curcuma longa L. (Zingiberaceae) that protect PC12 rat pheochromocytoma and normal human umbilical vein endothelial cells from betaA(1-42) insult. Neurosci Lett. 2001;303(1):57-61.

4. Simon A, Allais D, Duroux JL, Basly J, Durand-Fontanier S, Delage C. Inhibitory effect of curcuminoids on MCF-7 cell proliferation and structure-activity relationships. Cancer Lett. 1998;129(1):111-6.
5. Jayaprakasha GK, Jaganmohan Rao L, Sakariah KK. Antioxidant activities of curcumin, demethoxycurcumin and bisdemethoxycurcumin. Food Chemistry. 2006;98(4):720-4.

6. Sandur SK, Pandey MK, Sung B, Ahn KS, Murakami A, Sethi G, et al. Curcumin, demethoxycurcumin, bisdemethoxycurcumin, tetrahydrocurcumin and turmerones differentially regulate anti-inflammatory and anti-proliferative responses through a ROS-independent mechanism. Carcinogenesis. 2007;28(8):1765-73.

7. Chatterjee SN, Chakravarty AK, Yasmin H, Mazumder T. Comparison of efficacy of turmeric and commercial curcumin in immunological functions and gene regulation. International Journal of Pharmacology. 2009;5:333-45.

8. Račková L, Koštálová D, Bezáková L. Comparative study of two natural antioxidants, curcumin and Curcuma longa extract. Journal of Food and Nutrition Research. 2009;48:148-52.

9. Moon A, Ko EY. Natural products for chemoprevention of Breast cancer. J Cancer Prev. 2015;20:223-31.

10. Hajar Zamrus SN, Akhtar MN, Yeap SK, Quah CK, Loh WS, Alitheen SB, et al. Design, synthesis and cytotoxic effects of curcuminoids on HeLa, K562, MCF-7 and MDA-MB-231 cancer cell lines. Chemistry Central Journal. 2018;12(1):31.

11. Liu XP, Lv ZD, Zhao WJ, Dong Q, Li FN, Wang HB. Curcumin induces apoptosis in breast cancer cells and inhibits tumor growth in vitro and in vivo. Int $\mathrm{J}$ Clin Exp Pathol. 2014;7:2818-24.

12. Park SJ, Garcia CV, Shin GH, Kim JT. Improvement of curcuminoid bioaccessibility from turmeric by a nanostructured lipid carrier system. Food Chem. 2018;251:51-7.

13. Cuomo J, Appendino G, Dern AS, Schneider E, McKinnon T, Brown MJ, et al. Comparative absorption of a standardized curcuminoid mixture and its lecithin formulation. J Nat Prod. 2011;74(4):664-9.

14. Yeap YY, Trevaskis NL, Porter CJ. Lipid absorption triggers drug supersaturation at the intestinal unstirred water layer and promotes drug absorption from mixed micelles. Pharm Res. 2013;30(12):3045-58.

15. Stremmel W, Staffer S, Gan-Schreier H, Wannhoff A, Bach M, Gauss A. Phosphatidylcholine passes through lateral tight junctions for paracellular transport to the apical side of the polarized intestinal tumor cell-line $\mathrm{CaCo}$. Biochimica et Biophysica Acta (BBA) - Molecular and Cell Biology of Lipids. 2016;1861 (9, Part A):1161-9.

16. Thanki K, Gangwal R, Sangamwar AT, Jain S. Oral delivery of anticancer drugs: challenges and opportunities. J Control Release. 2013;170(1):15-40. 
17. Fei Yu YL, Chen Q, He Y, Wang H, Yang L, Guo S, et al. Monodisperse microparticles loaded with the self-assembled berberine-phospholipid complex-based phytosomes for improving oral bioavailability and enhancing hypoglycemic efficiency. European Journal of Pharmaceutics and Biopharmaceutics. 2016;103:136-48

18. Peng Q, Zhang ZR, Sun X, Zuo J, Zhao D, Gong T. Mechanisms of phospholipid complex loaded nanoparticles enhancing the oral bioavailability. Mol Pharm. 2010;7(2):565-75.

19. Kalepun S, Manthina M, Padavala V. Oral lipid-based drug delivery systems - an overview. Acta Pharmaceutica Sinica B. 2013;3(6):361-72.

20. Raveendran R, Bhuvaneshwar G, Sharma C. In vitro cytotoxicity and cellular uptake of curcumin-loaded Pluronic/Polycaprolactone micelles in colorectal adenocarcinoma cells. J Biomater Appl. 2013;27(7):811-27.

21. Mohanraj VJ, Chen Y. Nanoparticles - A Review. Tropical Journal of Pharmaceutical Research. 2006;5(1):561-73

22. Torchilin V. Tumor delivery of macromolecular drugs based on the EPR effect. Adv Drug Deliv Rev. 2011;63(3):131-5.

23. Karp JM, Peer D, Hong S, Farokhzad OC, Margalit R, Langer R. Nanocarriers as an emerging platform for cancer therapy. Nat Nanotechnol. 2007;2(12):751-60.

24. Li $Y$, Wu H, Yang $X$, Jia M, Li $Y$, Huang $Y$, et al. Mitomycin C-soybean phosphatidylcholine complex-loaded self-assembled PEG-lipid-PLA hybrid nanoparticles for targeted drug delivery and dual-controlled drug release. Mol Pharm. 2014;11(8):2915-27.

25. Solmaz Rasaie SG, Mohammadi M, Hamishehkar H. Nano phytosomes of Quercetin: A promising formulation for fortification of food products with antioxidants. Pharmaceutical Sciences. 2014;20:96-101.
26. Lee R, Cambrea Kalani J, Seu R, Everly M, Hovis JS. Influence of lipid chemistry on membrane fluidity: Tail and headgroup interactions. Biophys $\mathrm{J}$ 2006;91(10):3727-35.

27. Tripp $\mathrm{CP}$, Chen $\mathrm{CF}$. An infrared spectroscopic based method to measure membrane permeance in liposomes. Biochimica et Biophysica Acta (BBA) Biomembranes. 2008;1778(10):2266-72.

28. Bhattacharya S, Haldar S. Interactions between cholesterol and lipids in bilayer membranes. Role of lipid headgroup and hydrocarbon chain-backbone linkage. Biochimica et Biophysica Acta (BBA) - Biomembranes. 2000;1467(1):39-53.

29. Brian Cannon GH, Huang J. Time-resolved fluorescence and fourier transform infrared spectroscopic investigations of lateral packing defects and superlattice domains in compositionally uniform cholesterol/phosphatidylcholine bilayers. Biophysical Journal. 2003;84:3777-91.

30. Xie J, Li Y, Song L, Pan Z, Ye S, Hou Z. Design of a novel curcumin-soybean phosphatidylcholine complex-based targeted drug delivery systems. Drug Deliv. 2017;24(1):707-19.

31. Li HQ, Jin LJ, Wu FF, Li XY, You JS, Cao ZH, et al. Effect of curcumin on proliferation, cell cycle, and caspases and MCF-7 cells. African Journal of Pharmacy and Pharmacology. 2012.

32. ThakkarVR, Patel PB, Patel JS. Cellular effect of curcumin and citral combination on breast cancer cells: Induction of apoptosis and cell cycle arrest. J Breast Cancer. 2015;18(3):225-34.

33. Lu M, Qiu Q, Luo X, Liu X, Sun J, Wang C, et al. Phyto-phospholipid complexes (phytosomes): A novel strategy to improve the bioavailability of active constituents. Asian Journal of Pharmaceutical Sciences. 2018.

Cite this article: Long NV, Thu Ha BT, Tuan AV, Luong HV, Linh NT, Duc TC, et al. Phytosomal Nanoparticles Preparation of Curcuminoids to Enhance Cellular Uptake of Curcuminoids on Breast Cancer Cell Line MCF-7. Pharmacog J. 2019;11(5):1037-45. 